\title{
Perioperative Medizin großer viszeralchirurgischer Eingriffe
}

\author{
Elke Muhla Wolfgang H. Hartl \\ ${ }^{a}$ Klinik für Chirurgie, Universitätsklinikum Schleswig-Holstein, Campus Lübeck, \\ ${ }^{\mathrm{b}}$ Chirurgische Klinik und Poliklinik der Universität, Campus Grosshadern, Ludwig-Maximilians-Universität, München, Deutschland
}

Große viszeralchirurgische Eingriffe sind nicht nur eine Herausforderung für den Chirurgen als Operateur, sondern auch für den Chirurgen als perioperativ tätigen Arzt und für den operativen Intensivmediziner. Die Erweiterung der operationstechnischen Möglichkeiten zur Minimierung des operativen Traumas (minimal invasive Chirurgie) und die Entwicklung in der Intensivmedizin in den letzten Jahren haben Operationen ermöglicht, die vor mehr als zehn Jahren noch undenkbar waren. So kommen immer komplexer vorerkrankte und immer ältere Patienten zu immer komplexeren Eingriffen in unsere Kliniken.

Zur perioperativen Medizin in der Viszeralchirurgie (und auch in allen anderen chirurgischen Gebieten) gehören neben der präoperativen Risikoabschätzung, der Patientenkonditionierung und -vorbereitung sowie der differenzierten Indikationsstellung auch die postoperative Überwachung, Komplikationsvermeidungsstrategien und das Komplikationsmanagement. Sie beinhaltet unter anderem Schmerztherapie, Ernährungstherapie, Therapie der Sepsis, Volumenmanagement, Antibiotikatherapie, Gerinnungsmanagement und Thromboseprophylaxe, aber auch die Berücksichtigung diverser meist internistischer - Begleiterkrankungen und zudem eingriffsspezifische Behandlungskonzepte. Einige dieser Themen werden im vorliegenden Heft zur perioperativen Medizin viszeralchirurgischer Eingriffe behandelt.

Die perioperative Medizin leistet einen zunehmend wichtigen Beitrag in der Behandlung chirurgischer Patienten - präoperativ auf den normalen Pflegestationen, postoperativ auch auf den Intensivstationen. Der Chirurg muss die Herausforderungen der perioperativen Medizin annehmen. Wenn wir als Chirurgen dieses Arbeitsfeld nicht kompetent besetzen, werden es andere tun (müssen).

Die obligatorische sechsmonatige Weiterbildung in der Intensivmedizin im Rahmen der Weiterbildung in einem chir- urgischen Fach dient unter anderem auch dazu, Ärzte für dieses Tätigkeitsfeld fit zu machen. Ob diese Weiterbildungszeit dafür ausreicht, erscheint allerdings fraglich angesichts der Komplexität der perioperativen Probleme unserer Patienten. Eine Veränderung der Weiterbildungsstruktur sollte in den Fachgesellschaften und Berufsverbänden sowie in den Ärztekammern diskutiert und angegangen werden:

Nur wenige Chirurgen absolvieren die zweijährige Zusatzweiterbildung «Intensivmedizin», auch weil Karriereperspektiven fehlen und die Arbeitsbedingungen durch Schichtdienst und Personalmangel wenig attraktiv sind. Die Chirurgie hat ein Nachwuchsproblem, die chirurgische Intensivmedizin noch viel mehr - und zum Teil auch die Intensivmedizin in anderen Fächern. Ein Lösungsansatz könnte die Umwandlung der Zusatzweiterbildung «Intensivmedizin» in einen Schwerpunkt Intensivmedizin in den Fächern Anästhesiologie, Chirurgie, Innere Medizin, Kinder- und Jugendmedizin und Neurologie/Neurochirurgie sein.

Als weiterer Baustein innerhalb der Weiterbildungsmöglichkeiten ist eine neue Zusatzweiterbildung «Perioperative Medizin» denkbar für alle chirurgischen Fachgebiete mit Zugang zur Weiterbildung «Intensivmedizin» (also für Allgemein- und Viszeralchirurgie, Herz- und Gefäßchirurgie, Thoraxchirurgie, Unfallchirurgie, Neurochirurgie und Plastische Chirurgie/Verbrennungsmedizin). Für den Erwerb einer solchen Zusatzweiterbildung sollte eine mindestens einjährige intensivmedizinische Weiterbildung Voraussetzung sein.

Die Weiterbildung sollte fokussiert sein auf die Versorgung von chirurgischen Patienten auf der Normalstation, auf ihre metabolischen, internistischen, kardiologischen, pulmonologischen und neurologischen Begleiterkrankungen, Ernährung, Schmerztherapie und auf adjuvante medikamentöse Therapien im perioperativen Kontext. Die so weitergebilde-

\section{KARGER \\ Fax +497614520714 \\ Information@Karger.de}

www.karger.com (c) 2011 S. Karger GmbH, Freiburg

$1662-6664 / 11 / 0271-0009 \$ 38.00 / 0$

Accessible online at:

www.karger.com/vim
Prof. Dr. Elke Muhl

Klinik für Chirurgie, Universitätsklinikum Schleswig-Holstein Campus Lübeck

Ratzeburger Allee 160, 23538 Lübeck, Deutschland

Tel. +49 451 500-2025, Fax -2026

Elke.muhl@uk-sh.de 
ten Ärzte sollten Diagnostik, Therapie und Prävention von Organdysfunktionen und Stoffwechselstörungen sowie Risikostratifizierung und Komplikationsmanagement für operative Patienten beherrschen. Ärzte mit dieser Weiterbildung könnten im Stationsdienst auf den Normalstationen der Chirurgie ihr Tätigkeitsfeld finden. Im perioperativen Arbeitsfeld sind - besser als in der überwiegend operativen Tätigkeit und in Arbeitsfeldern mit einem hohen Anteil an Notfallversorgung - Teilzeitarbeitsplätze mit verlässlichen Arbeitszeiten möglich, die von Ärzten und Ärztinnen mit kleinen Kindern zunehmend nachgefragt werden. Eine spezielle Weiterbildung in perioperativer Medizin könnte auch dem überwiegend operativ tätigen Chirurgen in der ganzheitlichen Versorgung seiner Patienten nützlich sein.

Die Qualität der perioperativen Versorgung ist ein - vielleicht noch zu wenig beachtetes - Qualitätsmerkmal einer chirurgischen Klinik und von großer Bedeutung für das Outcome chirurgischer Patienten. 